\title{
A!
}

This is an electronic reprint of the original article.

This reprint may differ from the original in pagination and typographic detail.

Kutvonen, A.; Ala-Nissila, T.; Pekola, J.

\section{Entropy production in a non-Markovian environment}

Published in:

Physical Review E

DOI:

10.1103/PhysRevE.92.012107

Published: 06/07/2015

Document Version

Publisher's PDF, also known as Version of record

Please cite the original version:

Kutvonen, A., Ala-Nissila, T., \& Pekola, J. (2015). Entropy production in a non-Markovian environment. Physical Review E, 92(1), 1-7. [012107]. https://doi.org/10.1103/PhysRevE.92.012107

This material is protected by copyright and other intellectual property rights, and duplication or sale of all or part of any of the repository collections is not permitted, except that material may be duplicated by you for your research use or educational purposes in electronic or print form. You must obtain permission for any other use. Electronic or print copies may not be offered, whether for sale or otherwise to anyone who is not an authorised user. 


\title{
Entropy production in a non-Markovian environment
}

\author{
Aki Kutvonen, ${ }^{1}$ Tapio Ala-Nissila, ${ }^{1,2}$ and Jukka Pekola ${ }^{3}$ \\ ${ }^{1}$ COMP Center of Excellence, Department of Applied Physics, Aalto University School of Science, \\ P.O. Box 11000, FI-00076 Aalto, Espoo, Finland \\ ${ }^{2}$ Department of Physics, Brown University, Providence, Rhode Island 02912-1843, USA \\ ${ }^{3}$ Low Temperature Laboratory (OVLL) and Department of Applied Physics, Aalto University School of Science, \\ P.O. Box 13500, FI-00076 Aalto, Espoo, Finland
}

(Received 11 August 2014; revised manuscript received 26 March 2015; published 6 July 2015)

\begin{abstract}
Stochastic thermodynamics and the associated fluctuation relations provide the means to extend the fundamental laws of thermodynamics to small scales and systems out of equilibrium. The fluctuating thermodynamic variables are usually treated in the context of either isolated Hamiltonian evolution, or Markovian dynamics in open systems. However, there is no reason a priori why the Markovian approximation should be valid in driven systems under nonequilibrium conditions. In this work, we introduce an explicitly non-Markovian model of dynamics of an open system, where the correlations between the system and the environment drive a subset of the environment out of equilibrium. Such an environment gives rise to a new type of non-Markovian entropy production term. Such non-Markovian components must be taken into account in order to recover the fluctuation relations for entropy. As a concrete example, we explicitly derive such modified fluctuation relations for the case of an overheated single electron box.
\end{abstract}

DOI: 10.1103/PhysRevE.92.012107

PACS number(s): 05.70.Ln, 05.40.-a, 74.40.-n, 74.55.+v

The fundamental laws of thermodynamics are among the most powerful postulates of physics due to their universality. Motivated by recent developments in nanotechnology, a great effort has been made to extend thermodynamics to microscopically small systems and to nonequilibrium processes [1-4]. These efforts are commonly formulated in the form of stochastic thermodynamics, which culminates into fluctuation relations connecting extensive thermodynamic variables such as work, free energy, and entropy [5-9].

Stochastic thermodynamics and the associated fluctuation relations can be formulated through trajectory-dependent fluctuating quantities, typically assuming Markovian evolution for open systems interacting with an ideal heat bath. The Markovian assumption means that all the degrees of freedom of the infinite environment relax to the equilibrium state at time scales much faster than those of the system, and thus there are no memory effects caused by the coupling of the system and the environment. However, since by definition of an open system there is always a coupling term in the Hamiltonian of the total (isolated) system that contains both background and system degrees of freedom, Markovian evolution is, strictly speaking, an approximation. In small systems, there is no reason a priori for a division to clearly separate fast background and slow system degrees of freedom, and thus nonMarkovian effects such as memory of dynamics may become important.

Some recent works have examined entropy production under non-Markovian dynamics by considering different types of memory or by using other thermodynamic arguments [10-13]. However, the crucial assumption behind these models is that the environment cannot change during the process [13]. In this work, we consider a non-Markovian system in which some degrees of freedom in the environment are influenced by the interaction with the system, and thus the environment must be explicitly taken into account. We extend the classical stochastic thermodynamics approach to include the nonMarkovian dynamics arising from such effects.
In particular, we show how violation of the Markovian approximation of the environment leads to the generation of additional entropy. Our approach allows the identification and separation of the total entropy production into standard Markovian and new non-Markovian components. The existence of a non-Markovian component of entropy production leads to modifications of the standard fluctuation relations for entropy. As a concrete example of this, we consider the single-electron box (SEB), which has proven to be an excellent test bench for studying thermodynamics at small scales [14-18]. In particular, we demonstrate that an overheated SEB is an experimentally feasible system with well-defined non-Markovian dynamics, and we derive fluctuation relations for entropy production in such systems.

We follow the standard approach of stochastic thermodynamics (see, e.g., $[5,19]$ and references therein) and consider a system whose degrees of freedom evolve under the influence of a time-dependent control parameter $\lambda(t)$ from $x_{0}=x(0)$ to $x_{f}=x\left(t_{f}\right)$ along a trajectory $x(t)$. The time reverse processes are given by $\tilde{x}(t)=x\left(t_{f}-t\right)$ and $\tilde{\lambda}(t)=\lambda\left(t_{f}-t\right)$. In stochastic thermodynamics, the total entropy generation of any given trajectory $x(t)$ is defined as

$$
\Delta S_{T}=\Delta S_{S}+\Delta S_{F}=\ln \frac{p_{I}\left[x_{0}\right]}{p_{F}\left[\tilde{x}_{0}\right]}+\ln \frac{p\left[x(t) \mid x_{0}\right]}{\tilde{p}\left[\tilde{x}(t) \mid \tilde{x}_{0}\right]}
$$

where $\Delta S_{S}$ is the change in the system entropy and $\Delta S_{F}$ is the entropy flow to the environment (or medium entropy generation), $p\left[x(t) \mid x_{0}\right]$ and $\tilde{p}\left[\tilde{x}(t) \mid \tilde{x}_{0}\right]$ are the probabilities of the forward and reverse path, and $p_{I}\left[x_{0}\right]$ and $p_{F}\left[\tilde{x}_{0}\right]$ are the probabilities of the initial and final states, respectively. From the conservation of probability, it immediately follows that

$$
\left\langle e^{-\Delta S_{T}}\right\rangle=1
$$


If the dynamics are stochastic and governed by a Markovian master equation, the entropy flow $\Delta S_{F}$ can be expressed as

$$
\Delta S_{F}[x]=\ln \prod_{j=1}^{N} \frac{W_{x_{j}, x_{j-1}}\left(\lambda_{j}\right)}{W_{x_{j-1}, x_{j}}\left(\lambda_{j}\right)},
$$

where $W_{x_{j}, x_{j-1}}\left(\lambda_{j}\right)$ is the transition rate from $x_{j-1}=x\left(t_{j-1}\right)$ to $x_{j}$ at control parameter value $\lambda_{j}=\lambda\left(t_{j}\right)$. Furthermore, if the rates are connected by the local detailed balance condition (LDB)

$$
\frac{W_{x_{j}, x_{j-1}}\left(\lambda_{j}\right)}{W_{x_{j-1}, x_{j}}\left(\lambda_{j}\right)}=e^{\beta Q_{j}}
$$

where $Q_{j}$ is the heat dissipation from the system to the bath at $t=t_{j}$ and $\beta=1 / T_{B}$ is the inverse temperature of the heat bath, the entropy flow becomes

$$
\Delta S_{F}[x]=\Delta S_{F}^{m}[x]=\beta Q[x],
$$

where $Q=\sum_{j} Q_{j}$ is the heat flow from the system during trajectory $x$. We note that if the initial distribution $p_{I}$ is that of a canonical equilibrium state, the total entropy is given by the dissipated work $\Delta S_{T}=W[x]-\Delta F$, and Eq. (1) becomes the Crooks fluctuation relation and Eq. (2) the Jarzynski equality $[7,9]$.

The Markovian approximation, where the rate matrix is only a function of $\lambda$ [cf. Eq. (3)], and the LDB condition of Eq. (5) together require that the environment is an ideal heat bath with a relaxation time $\tau_{B}$ much shorter than any other time scale in the total system. However, since there is always a finite interaction between the system and the environment, there are always some correlations between their dynamics. In small systems, such correlations may become important if there is no clear separation of time scales into a fast, infinitely large environment and a slow, finite system. We now consider a setup beyond the Markovian assumption, namely the case in which the environment as a whole is not an ideal heat bath described by equilibrium temperature $T_{B}$. We assume that there exists a subset of degrees of freedom of the environment that are correlated with the dynamics of the system and do not relax to equilibrium defined by $T_{B}$ on a time scale faster than that of the system. We call these degrees of freedom the nonequilibrium subsystem (NE) of the environment, which cannot be described by the bath's thermal equilibrium distribution during the drive and must therefore be explicitly taken into account.

In general, a rigorous study of such nonequilibrium fluctuations in the environment requires full knowledge of the time evolution of all the degrees of freedom inside the NE. However, if it has an internal relaxation time $\tau_{\mathrm{NE}}$ much shorter than the characteristic relaxation time scale of the system $\tau_{S}$, we can simplify the problem. In addition, we assume that the $\mathrm{NE}$ is weakly coupled to the rest of the environment such that all the degrees of freedom in the NE mutually relax to a (nonequilibrium) state at time scales $\tau_{\mathrm{NE}} \ll \tau_{S}$. We note that even if the relaxation inside the NE is the fastest time scale, Markovian evolution does not follow here since the NE is finite, and due to energy deposition from the system during the trajectory $x$, the NE will evolve through quasiequilibrium states different from the equilibrium state of the rest of the environment. That is to say, the transition $x_{i} \rightarrow x_{i+1}$ is affected by the earlier transitions $\left\{x_{j} \rightarrow x_{j+1}\right\}$, where $j<i$.
We will further assume that the NE contains sufficiently many degrees of freedom such that it can be described by a time-dependent probability distribution function (density operator) $\rho_{\mathrm{NE}}(t)$ with an effective temperature $T(t)$. Furthermore, as the relaxation time $\tau_{B}$ is short and the correlation between the NE and the rest of the environment is weak, we assume that the environment outside of the NE is described by the equilibrium distribution $\rho^{\mathrm{eq}}$ and acts like a Markovian heat bath. In this case, the total distribution $\rho_{T}(t)$ can be generally written as $\rho_{T}(t)=\rho_{S ; \mathrm{NE}}(t) \otimes \rho^{\mathrm{eq}}$, where $\rho_{S ; \mathrm{NE}}(t)$ is the joint probability distribution of the system and the NE. Further, since we assume that $\tau_{\mathrm{NE}} \ll \tau_{S}$, we can separate this joint distribution function into its two constituents and write the total distribution as

$$
\rho_{T}(t)=\rho_{S}(t) \otimes \rho_{\mathrm{NE}}(t) \otimes \rho^{\mathrm{eq}} .
$$

As a result, the total trajectory entropy $\Delta S_{T}$ is no longer given by its Markovian component $\Delta S_{T}^{m}=\Delta S_{F}^{m}+\Delta S_{S}$. Instead, we have an additional source of entropy, $S_{\mathrm{NE}}(t)=-\ln \rho_{\mathrm{NE}}$, coming from the fact that in addition to $\rho_{S}$, the probability distribution $\rho_{\mathrm{NE}}(t)$ changes in time.

Returning to the dynamics of the system, the time scale separation assumed here means that the system is effectively driven in an environment controlled by the $\mathrm{NE}$ at an effective temperature $T(t)$ instead of the bath temperature $T_{B}$. Therefore, the transition rates no longer satisfy the LDB condition of Eq. (4), but instead they should satisfy the condition

$$
\frac{W_{x_{j}, x_{j-1}}\left(\lambda_{j}\right)}{W_{x_{j-1}, x_{j}}\left(\lambda_{j}\right)}=e^{\left(\beta+\Delta \beta_{j}\right) Q_{j}},
$$

where $\Delta \beta_{j} \equiv 1 / T\left(t_{j}\right)-1 / T_{B}$. As long as the total system is described by Eq. (6) between the transitions, the NE can be integrated out giving rise to transition rates for the system states in Eq. (7). However, for example, if the number of degrees of freedom in the NE is not large enough, $\Delta \beta_{j}$ may not be associated with any physical temperature, but it should be defined through Eq. (7) as a parameter modifying the LDB condition. Assuming Eq. (7) holds and integrating out the equilibrium part gives the associated entropy flow $\Delta S_{F}$ [Eq. (3)] as

$$
\Delta S_{F}=\Delta S_{F}^{m}+\Delta S_{F}^{n m}=\beta Q+\Delta \beta Q,
$$

where now an additional non-Markovian contribution $\Delta S_{F}^{n m}$ appears in addition to the Markovian component $\Delta S_{F}^{m}=\beta Q$; cf. Eq. (5). We note that even if the entropy flow can always be mathematically written as in Eq. (8), the physical interpretation of $\triangle \beta Q$ in the general case may not be clear.

Since the heat bath and the NE are coupled, there can also be heat flow $Q^{B}$ from the NE to the bath. The associated entropy component is given by

$$
\Delta S_{F}^{B}=\beta Q^{B} .
$$

Adding the non-Markovian sources of entropy production to $\Delta S_{T}$, we can conclude that the total entropy production in now given by

$$
\Delta S_{T}=\Delta S_{S}+\Delta S_{F}^{m}+\Delta S_{F}^{n m}+\Delta S_{\mathrm{NE}}+\Delta S_{F}^{B},
$$

where the sum of the first two terms is the Markovian component of the entropy production $\Delta S_{T}^{m}$, and the sum of 


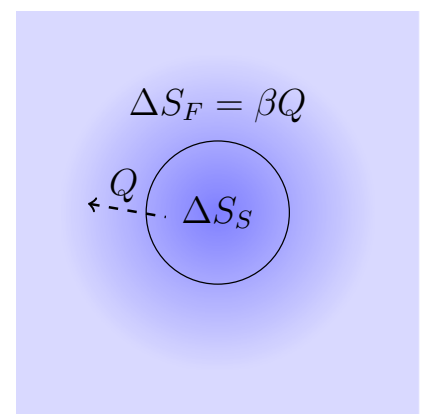

$$
\rho_{S}(t) \otimes \rho^{e q}
$$

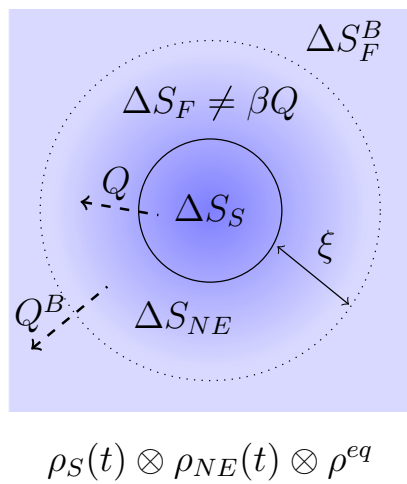

FIG. 1. (Color online) A schematic of the comparison between entropy generation in the two cases considered in the text. The left panel illustrates a purely Markovian system, in which the environment is assumed to stay in equilibrium during the process. The right panel shows the case of an NE, with a measure $\xi$ that now constitutes a subset of the bath degrees of freedom. The NE can be driven away from equilibrium due to the coupling to the system, leading to an additional entropy production (see text for details).

the last three terms is the non-Markovian counterpart $\Delta S_{T}^{n m}$. The different sources of entropy production are schematically illustrated in Fig. 1.

To make the arguments presented above concrete, we next proceed to consider a physically realistic example, namely the single-electron box (SEB) [20], where single-electron transitions are externally induced between two metallic islands. In recent experiments, various fluctuation relations have been measured on a SEB [14,17], and it has been harnessed for experiments to convert information into energy (Maxwell's demon) in Refs. $[15,16]$. The SEB is particularly suitable for studies of nonequilibrium statistical phenomena due to the limited number of relevant degrees of freedom, the clear separation of different time scales, and well-defined and easily measured energy scales.

In our case, the SEB is composed of a metallic island coupled to a metallic electrode (lead) by a tunnel junction and to another electrode by a capacitor; see Fig. 2. The relevant part of the Hamiltonian of the system is that of its Coulomb energy, given by $H_{S}\left(n, n_{g}\right)=E_{C}\left(n-n_{g}\right)^{2}$, where $E_{C}=e^{2} /\left(2 C_{\Sigma}\right), e$ is the electron charge, and $C_{\Sigma}=C+C_{g}+C_{0}$ is the total capacitance, which is given by the sum of those of the tunnel junction $C$, the gate capacitance $C_{g}$, and the self-capacitance $C_{0}$ of the island. The constant $E_{C}$ gives the unit of energy to charge the box by an extra electron. The control parameter $n_{g}=C_{g} V_{g} / e$ is given by the scaled gate voltage $V_{g}$ driving the electrons between the islands. The stochastic variable, $n$, is the number of extra electrons on the island of the box and should be considered as the system variable. Note, however, that in a metallic SEB, the total number of electrons is large, of the order of $10^{9}$, whereas $n$ describes the deviation from the particular equilibrium number on the island. In a well-controlled experiment, $n$ can assume only two values, say $n=0$ and 1 . To achieve this regime, the SEB needs to be operated at low enough temperatures, $k_{B} T<E_{C}$, and the values of the control parameter $n_{g}$ vary within one period (amplitude $<1$ ) only. A detailed discussion on the energetics

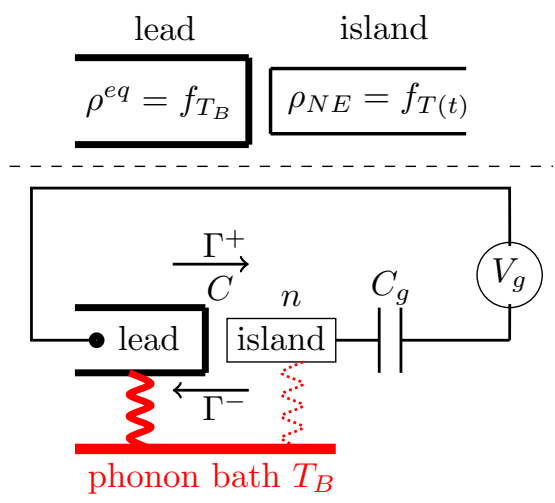

FIG. 2. (Color online) Schematic of a single-electron box. The number of excess electrons on the island, $n$, is a stochastic variable governed by the tunneling rates to $\left(\Gamma^{+}\right)$and from $\left(\Gamma^{-}\right)$the island, which depend on the control parameter $V_{G}$, which denotes the gate voltage. As the island has a finite heat capacity, and since it is weakly coupled to the substrate, the electrons on the island tend to occupy a Fermi distribution $f_{T}(t)$ at an effective temperature $T(t)$ that differs from the bath temperature. The other side of the tunnel junction is a reservoir with essentially infinite heat capacity and strong coupling to the phonon bath, and thus the electrons in this lead are Fermidistributed at the equilibrium temperature $T_{B}$.

and the different thermodynamic variables in the SEB can be found in Refs. [21,22].

In the present case, we consider the SEB in an experimentally feasible situation, where the metallic island is small and thus the heat capacity of the island is finite [18]. The collective of $10^{9}$ electrons on the island forms the NE of the setup, which can be driven away from equilibrium due to the small size of the metallic island. The relaxation of nonequilibrium excitations created by energy deposition of a tunneling electron (electron-electron relaxation rate) on both sides of the tunnel junction is the fastest time scale in the problem $\left(\tau_{\mathrm{NE}}\right)$. In experiments, the internal relaxation time $\tau_{\mathrm{NE}}$ is of order of $10^{-9} \mathrm{~s}$, while the relaxation time back to the equilibrium by the coupling to the phonon bath of the substrate on which the SEB is fabricated is of order of $10^{-4} \mathrm{~s}$. Therefore, the electrons on the small metallic island of the tunnel junction adopt a Fermi distribution at a different effective temperature during the drive. We note that this temperature is a result of the stochastic energy deposition from tunneling electrons on the small island due to the drive by the control gate. The state of the whole system is thus described by a product form of type $\rho_{T}(t)=\rho_{S}^{n}(t) \otimes f_{T(t)}\left(E_{I}\right) \otimes f_{T_{B}}(E)$ [cf. Eq. (6)], where $\rho_{S}^{n}(t)$ is the distribution of excess electrons $n$ on the island at time $t$, and $f_{T(t)}\left(E_{I}\right)$ is the Fermi distribution of electron energy $E_{I}$ on the island at an effective temperature $T(t)$, which depends on the energies of the previous tunneling events. Therefore, the process is non-Markovian. Finally, $f_{T_{B}}(E)$ is the Fermi distribution on the lead side of the tunnel junction at the equilibrium temperature $T_{B}$. Thus the SEB setup here is a concrete, physical realization of a well-defined NE, as discussed in the general theoretical setting. More details on the operation of an SEB in this regime can be found in Refs. [18,23]. 
We next proceed to study dissipation in a tunneling process. Standard Fermi Golden Rule calculations yield the tunneling rates

$$
\Gamma^{ \pm}(t)_{T(t)}=\int_{-\infty}^{\infty} \gamma_{T(t)}^{ \pm}(E, t) d E
$$

where + denotes tunneling in, - denotes tunneling out, and $\gamma_{T(t)}^{ \pm}(E, t) d E$ is the differential electron tunneling rate within the energy interval $d E$ around $E$ (from the Fermi level of the lead) such that

$$
\gamma_{T(t)}^{ \pm}(E, t)=\frac{1}{e^{2} R_{T}} f_{T_{B}}( \pm E)\left(1-f_{T(t)}\{ \pm[E+\Delta U(t)]\}\right)
$$

where $R_{T}$ is the tunneling resistance and $\Delta U$ is the chemical potential difference across the junction. In equilibrium, the rates $\Gamma^{ \pm}$are LDB connected, but if $T(t) \neq T_{B}$, the LDB condition is broken, and a condition of the type Eq. (7) holds.

In a tunneling event, the total dissipated heat is given by

$$
Q_{\mathrm{tot}}^{ \pm}=Q_{I}^{ \pm}+Q_{L}^{ \pm}= \pm \Delta U
$$

where the heat deposited to the lead in a tunneling event is given by $Q_{L}^{ \pm}=\mp E$ and the heat deposited to the island is $Q_{I}^{ \pm}= \pm(E+\Delta U)$. Equivalently, the total dissipated heat in a tunneling (in) event is the energy released by the system, $\Delta U=H_{S}\left(0, n_{g}\right)-H_{S}\left(1, n_{g}\right)=E_{C}\left(2 n_{g}-1\right)$.

Since in a tunneling event an energy $Q_{I}^{ \pm}$is deposited to local temperature $T(t)$ and energy $Q_{L}^{ \pm}$to temperature $T_{B}$, we can write the entropy flow in a tunneling event as

$$
S_{F}^{ \pm}=(\beta+\Delta \beta) Q_{I}^{ \pm}+\beta Q_{L}^{ \pm},
$$

where the non-Markovian component is given by $S_{F}^{n m, \pm}=$ $\Delta \beta Q_{I}^{ \pm}, \beta=1 / k_{B} T_{B}$, and $\Delta \beta \equiv 1 / k_{B} T-1 / k_{B} T_{B}$. Another contribution to the non-Markovian sources is the entropy of the NE, i.e., the electron population of the island, given by $S_{I}=$ $-\ln f_{T}$, where $f_{T}$ is the probability distribution of degrees of freedom inside the NE $\left(\rho_{\mathrm{NE}}\right)$, the Fermi function of the island at temperature $T$. In a tunneling event at time $t$, an electron with energy $E$, which changes the temperature of the island from $T_{i}$ to $T_{i+1}$, induces an entropy change of

$$
S_{I}^{ \pm}=\log \frac{f_{T_{i}}\{ \pm[E+\Delta U(t)]\}}{f_{T_{i+1}}\{ \pm[E+\Delta U(t)]\}} .
$$

The total change of $S_{F}$ and $S_{I}$ in a trajectory is given by the sum over individual tunneling events [Eqs. (14) and (15)], which we denote by $\Delta S_{F}$ and $\Delta S_{I}$, respectively.

The standard trajectory system entropy change is given by

$$
\Delta S_{S}=\log \frac{p_{I}\left[n_{I}\right]}{p_{F}\left[n_{F}\right]},
$$

where $p_{I}\left(n_{I}\right)$ and $p_{F}\left(n_{F}\right)$ are the probabilities to sample the initial and final states $n_{I}$ and $n_{F}$, respectively.

Initially, the $\mathrm{NE}$ is coupled to the bath, and the initial temperature $T_{I}$ is sampled from distribution $p_{I}\left(T_{I}\right)$, which is in equilibrium normally distributed with a variance $k_{B} T^{2} / C$, where $C$ is the heat capacity of the island. Thus, in addition to the tunneling events, $S_{I}$ can change due to the heat transfer from the bath. The associated entropy is given by

$$
\Delta S_{I}^{T}=\ln \frac{p_{I}^{T}\left(T_{I}\right)}{p_{F}^{T}\left(T_{F}\right)}
$$

where $p_{F}\left(T_{F}\right)$ is the probability to sample the final temperature $T_{F}$. In typical experiments, the heat capacity of the small metallic island is of the order of $1000 k_{B}$, and the operating temperature is around $100 \mathrm{mK}$, resulting in initial temperature fluctuations of the order of a few $\mathrm{mK}$. Depending on the drive protocol, particularly the speed of the drive, the heat dissipation per tunneling electron is of the order of $k_{B}$, resulting in temperature fluctuations of the order of $0.1 \mathrm{mK}$ per tunneling event. We note that the electron-phonon coupling can be neglected at short time scales of the process such that we can neglect the term $\Delta S_{F}^{B}$ in Eq. (10).

Proceeding to the dynamics of the SEB, the probability that there exist no tunneling-in $(+)$ or tunneling-out $(-)$ events from $t_{i}$ to $t_{i+1}$, while the temperature of the island is $T$, is given by

$$
P_{N T}^{ \pm}\left[t_{i}, t_{i+1}, T\right]=e^{-\int_{t_{i}}^{t_{i+1}} \Gamma_{T}^{ \pm}\left(t^{\prime}\right) d t^{\prime}} .
$$

The probability that an electron with energy $E$ at time $t$ tunnels is given by $\gamma_{T}^{ \pm}(E, t)$. We denote the direction of tunneling event $i$ in a realization of $n$ tunneling events as $i_{n} \in\{+,-\}$. Because of the two possible states, $(i+1)_{n}=-i_{n}$. Furthermore, the time labeling of temperature $T(t)$ is chosen such that $T_{I}=$ $T\left(t_{0}\right)$, and at tunneling $i$ at time $t_{i}$ the temperature is $T\left(t_{i-1}\right)$. The tunneling event at $t_{i}$, where a heat $Q_{I}= \pm\left(E-\mu_{I}\right)$ is deposited to the island, changes the temperature of the island to $T\left(t_{i}\right)=T\left(t_{i-1}\right)+Q_{I} / C$. Thus the probability of a realization of a path $X$ is given by

$$
\begin{aligned}
P(X) \equiv & p_{I}\left[n_{I}\right] p_{I}^{T}\left(T_{I}\right) P_{N T}^{1_{n}}\left[0, t_{1}, T_{I}\right] \\
& \times \prod_{i=1}^{i=n} \gamma_{T\left(t_{i-1}\right)}^{i_{n}}\left(E_{i}, t_{i}\right) P_{N T}^{(i+1)_{n}}\left[t_{i}, t_{i+1}, T\left(t_{i}\right)\right],
\end{aligned}
$$

where $t_{n+1}=t_{f}$ is the final time of the process and $t_{0}=0$ is the initial time. The sum of probabilities of all possible paths is normalized, i.e.,

$$
\int P(X) d X \equiv \sum_{n_{I}} \sum_{\left\{i_{n}\right\}} \prod_{i=0}^{n} \int_{t_{i}}^{t_{i+1}} \int_{-\infty}^{\infty} P(X) d t_{i} d E_{i}=1 .
$$

The probability of a time-reversed trajectory $P_{R}\left(X_{R}\right)$ is the probability to observe the time-reversed trajectory $X_{R}$ of trajectory $X$ under time reversal of the external control $\lambda(t) \rightarrow \lambda\left(t_{f}-t\right)$. The trajectory $X_{R}$ starts from the final state of trajectory $X$. In addition, all the tunneling events are reversed. Thus we can write

$$
\begin{aligned}
P_{R}\left(X_{R}\right) \equiv & p_{F}\left[n_{F}\right] p_{F}^{T}\left[T_{F}\right] P_{N T}^{(n+1)_{n}}\left[t_{n}, t_{f}, T_{F}\right] \\
& \times \prod_{i=1}^{i=n} \gamma_{T\left(t_{i}\right)}^{-i_{n}}\left(E_{i}, t_{i}\right) P_{N T}^{i_{n}}\left[t_{i-1}, t_{i}, T\left(t_{i-1}\right)\right]
\end{aligned}
$$

The probability $P_{R}\left(X_{R}\right)$ is also normalized to unity. An example of $P(X)$ and the corresponding $P_{R}\left(X_{R}\right)$ is illustrated in Fig. 3.

Using the relation between the forward and reverse paths, we show in the Appendix that the associated probabilities 

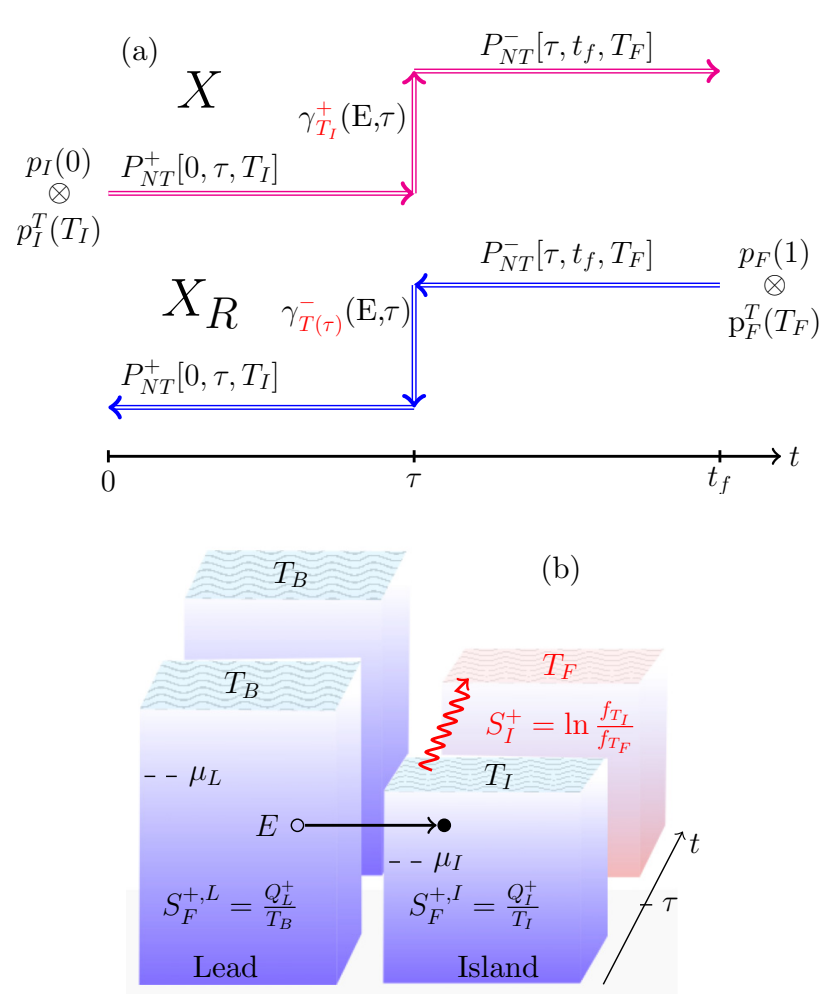

FIG. 3. (Color online) (a) An example of a trajectory $X$ starting from state $n=0, T(0)=T_{I}$ followed by a single tunneling-in event at time $t$ with energy $E$. Due to the relaxation of energy $Q_{I}^{+}=\mu_{I}-E$, the effective temperature on the island changes to $T_{F}$. The different terms denote the constituents of the total path probability. Trajectory $X_{R}$ demonstrates the reverse of $X$. The ratio of path probabilities yields $P(X) / P_{R}\left(X_{R}\right)=\exp \left[\Delta S_{T}[X]\right]$. (b) Entropy generation in a tunneling event, where heats $Q_{L}^{+}=\mu_{L}-E$ and $Q_{I}+=E-\mu_{I}$ are deposited to the lead and the island, respectively. The entropy flow in the event is given by $S_{F}^{+}=S_{F}^{+, L}+S_{F}^{+, I}=(\beta+\Delta \beta) Q_{\text {tot }}^{+}$. After the tunneling event at time $\tau$, the nonequilibrium excitation created by the tunneling electron relaxes. As a result, the electron energy distribution on the island changes from $f_{T_{I}}$ to $f_{T_{F}}$. The associated entropy change, the change of the NE entropy, is given by $S_{I}^{+}=$ $\ln \left[f_{T_{I}}(E+\Delta U) / f_{T_{F}}(E+\Delta U)\right]$.

satisfy the detailed fluctuation relation

$$
\frac{P(X)}{P_{R}\left(X_{R}\right)}=e^{\Delta S_{T}}=e^{\Delta S_{S}+\Delta S_{I}^{T}+\Delta S_{I}+\Delta S_{F}} .
$$

This is our first main result, which allows us to determine the total entropy production from the forward and reversed path probabilities. Furthermore, as we expect the total entropy production to satisfy the detailed fluctuation relation, Eq. (22) supports the claim that the non-Markovian sources of entropy production are given in a way we assumed. Using Eq. (22), we obtain the integral fluctuation relation

$$
\left\langle e^{-\Delta S_{T}}\right\rangle=\int e^{-\Delta S_{T}} P(X) d X=\int P_{R}\left(X_{R}\right) d X_{R}=1 .
$$

Furthermore, using the fluctuation relation for the Markovian entropy production $\Delta S_{T}^{m}=\Delta S_{S}+\Delta S_{F}^{m}$ yields

$$
\begin{aligned}
\left\langle e^{-\Delta S_{T}^{m}}\right\rangle & =\left\langle e^{-\Delta S_{T}+\Delta S_{T}^{n m}}\right\rangle=\int e^{-\Delta S_{T}[X]+\Delta S_{T}^{n m}[X]} P(X) d X \\
& =\int e^{-\Delta S_{T}^{n m}\left[X_{R}\right]} P_{R}\left(X_{R}\right) d X_{R}=\left\langle e^{-\Delta S_{T}^{n m}}\right\rangle_{R},
\end{aligned}
$$

where we used the fact that entropy generation is odd under time reversal, $\Delta S_{T}^{n m}\left[X_{R}\right]=-\Delta S_{T}^{n m}[X]$. By $\langle\cdot\rangle_{R}$ we denote an average over the process where the control parameter (gate voltage) protocol is time-reversed. In general, the right-hand side of Eq. (24) is not equal to unity. Thus, the standard fluctuation relations, which consider the entropy production only as a function of system degrees of freedom, are valid in the absence of entropy production $\Delta S_{T}^{n m}$. If the correlations extend to the environmental degrees of freedom, the environment has to be measured as well to determine the total entropy production.

To summarize, we have argued here that for any open system interacting with its environment, the interaction between the system and the environment unavoidably creates nonMarkovian correlations. If there is a clear time-scale separation between the slow system and the fast degrees of freedom of an ideal (infinite) environment, the Markovian approximation is acceptable. However, there are many systems in which this may not be the case. Here we have presented a quantitative way to take the non-Markovian effects into account by assuming that part of the environmental degrees of freedom form a nonequilibrium subsystem that remains in a nonequilibrium state while transitions in the system take place, and the rest of the environment remains in its thermal equilibrium state. In this formulation, the nonequilibrium state of the environment causing the memory effects is explicitly taken into account, allowing us to identify both Markovian and non-Markovian components of the total entropy production $\Delta S_{T}^{m}$ and $\Delta S_{T}^{n m}$, respectively. We have used the overheated SEB as an example, where this theoretical scenario is realized. The SEB allows a straightforward calculation and physical interpretation of the non-Markovian sources of entropy production. As our main results, we have derived detailed and integral fluctuation relations for the total entropy production within the SEB, and we showed how the Markovian and non-Markovian components of $\Delta S_{T}$ are related in terms of the forward and backward trajectories [Eq. (24)].

It is important to note that although our explicit calculations have been made for the SEB, the theoretical arguments in this article are generally valid for systems, where the state of the total system can be expressed as a product of the type of Eq. (6). Such systems include driven quantum-mechanical systems, where the total system is under unitary evolution and the initial state is modeled by a product state of the system and the environment density operators, $\hat{\rho}_{S}(t) \otimes \hat{\rho}_{E}^{\mathrm{eq}}$. In such systems the environment is driven as well, and as a result we expect additional non-Markovian entropy production [24-26].

This research has been supported in part by the Academy of Finland through its Centres of Excellence Programs (Projects No. 251748 and No. 250280). We wish to thank Carlos MejiaMonasterio, Elsi Laine, Samu Suomela, and Takahiro Sagawa for useful comments. 


\section{APPENDIX: DERIVATION OF EQ. (22) IN THE MAIN TEXT}

By implementing the definitions of forward and backward trajectories [Eqs. (19) and (21)], we obtain

$$
\frac{P(X)}{P_{R}\left(X_{R}\right)}=\frac{p_{I}\left[n_{I}\right] p_{I}^{T}\left(T_{I}\right) P_{N T}^{1_{n}}\left[0, t_{1}, T_{I}\right] \prod_{i=1}^{i=n} \gamma_{T\left(t_{i-1}\right)}^{i_{n}}\left(E_{i}, t_{i}\right) P_{N T}^{(i+1)_{n}}\left[t_{i}, t_{i+1}, T\left(t_{i}\right)\right]}{p_{F}\left[n_{F}\right] p_{F}^{T}\left[T_{F}\right] P_{N T}^{(n+1)_{n}}\left[t_{n}, t_{f}, T_{F}\right] \prod_{i=1}^{i=n} \gamma_{T\left(t_{i}\right)}^{-i_{n}}\left(E_{i}, t_{i}\right) P_{N T}^{i_{n}}\left[t_{i-1}, t_{i}, T\left(t_{i-1}\right)\right]} .
$$

The terms $P_{N T}$ cancel each other and the ratio of the terms $p_{I}$ and $p_{F}$ can be expressed with the use of Eqs. (16) and (17). Thus,

$$
\frac{P(X)}{P_{R}\left(X_{R}\right)}=e^{\Delta S_{S}+\Delta S_{I}^{T}} \prod_{i=1}^{i=n} \frac{\gamma_{T\left(t_{i-1}\right)}^{i_{n}}\left(E_{i}, t_{i}\right)}{\gamma_{T\left(t_{i}\right)}^{-i_{n}}\left(E_{i}, t_{i}\right)} .
$$

To simplify this expression, we first prove a local detailed balance type of equality,

$$
e^{-S_{F}^{ \pm}(E, t)} \gamma_{T}^{ \pm}(E, t)=\gamma_{T}^{\mp}(E, t) \text {. }
$$

By using the definition of $S_{F}^{ \pm}$[Eq. (14)], the left-hand side of the equation above yields

$$
e^{-S_{F}^{ \pm}(E, t)} \gamma_{T}^{ \pm}(E, t)=\frac{1}{e^{2} R_{T}} e^{ \pm \beta E} f_{T_{B}}( \pm E) e^{\mp(\beta+\Delta \beta)(E+\Delta U)}\left\{1-f_{T}[ \pm E+\Delta U(t)]\right\} .
$$

By using the properties of Fermi functions, $1-f_{T}(E)=f_{T}(-E)$ and $e^{(\beta+\Delta \beta)(E)} f_{T}(E)=f_{T}(-E)$, we obtain

$$
\frac{1}{e^{2} R_{T}} e^{ \pm \beta E} f_{T_{B}}( \pm E) e^{\mp(\beta+\Delta \beta)(E+\Delta U)}\left\{1-f_{T}[ \pm E+\Delta U(t)]\right\}=\frac{1}{e^{2} R_{T}} f_{T_{B}}(\mp E)\left\{1-f_{T}[\mp E+\Delta U(t)]\right\},
$$

which equals the right-hand side of Eq. (A3). Another result we need is

$$
\begin{aligned}
e^{-S_{I}^{ \pm}\left(t, E, T_{1}, T_{2}\right)} \gamma_{T_{1}}^{\mp}(E, t) & =\frac{f_{T_{2}}\{ \pm[E+\Delta U(t)]\}}{f_{T_{1}}\{ \pm[E+\Delta U(t)]\}} \frac{1}{e^{2} R_{T}} f_{T_{B}}(\mp E)\left\{1-f_{T_{1}}[\mp E+\Delta U(t)]\right\} \\
& =\frac{f_{T_{2}}\{ \pm[E+\Delta U(t)]\}}{f_{T_{1}}\{ \pm[E+\Delta U(t)]\}} \frac{1}{e^{2} R_{T}} f_{T_{B}}(\mp E)\left\{f_{T_{1}}[ \pm E+\Delta U(t)]\right\}=\frac{1}{e^{2} R_{T}} f_{T_{B}}(\mp E)\left\{1-f_{T_{2}}[\mp E+\Delta U(t)]\right\}
\end{aligned}
$$

where on the first line we used the definition of $S_{I}^{ \pm}$[Eq. (15)] and on the second line the Fermi function identity $1-f_{T}(E)=$ $f_{T}(-E)$. The right-hand side of Eq. (A6) equals $\gamma_{T_{2}}^{\mp}(E, t)$, thus

$$
e^{-S_{I}^{ \pm}\left(t, E, T_{1}, T_{2}\right)} \gamma_{T_{1}}^{\mp}(E, t)=\gamma_{T_{2}}^{\mp}(E, t)
$$

By combining Eqs. (A3) and (A7), we obtain

$$
\frac{\gamma_{T_{1}}^{ \pm}(E, t)}{\gamma_{T_{2}}^{\mp}(E, t)}=e^{S_{F}^{ \pm}+S_{I}^{ \pm}} .
$$

By using Eqs. (A2) and (A8) and the fact that the total entropy changes $\Delta S_{F}$ and $\Delta S_{I}$ are sums over the entropies $S_{F}^{ \pm}$and $S_{I}^{ \pm}$ produced in single events, we arrive at the final result,

$$
\frac{P(X)}{P_{R}\left(X_{R}\right)}=e^{\Delta S_{S}+\Delta S_{I}^{T}+\Delta S_{I}+\Delta S_{F}}=e^{\Delta S_{T}} .
$$

[1] C. Jarzynski, Annu. Rev. Condens. Matter Phys. 2, 329 (2011).

[2] U. Seifert, Rep. Prog. Phys. 75, 126001 (2012).

[3] C. Bustamante, J. Liphardt, and F. Ritort, Phys. Today 58(7), 43 (2005).

[4] C. B. D. Collin, F. Ritort, C. Jarzynski, S. B. Smith, and I. Tinoco, Nature (London) 437, 231 (2005).

[5] U. Seifert, Eur. Phys. J. B 64, 423 (2008).

[6] U. Seifert, Phys. Rev. Lett. 95, 040602 (2005).

[7] C. Jarzynski, Phys. Rev. E 56, 5018 (1997).

[8] T. Sagawa and M. Ueda, Phys. Rev. Lett. 104, 090602 (2010).

[9] G. E. Crooks, Phys. Rev. E 60, 2721 (1999).

[10] H. Hasegawa, Phys. Rev. E 84, 051124 (2011).
[11] R. García-García, Phys. Rev. E 86, 031117 (2012).

[12] M. Campisi, P. Hänggi, and P. Talkner, Rev. Mod. Phys. 83, 771 (2011).

[13] T. Speck and U. Seifert, J. Stat. Mech. (2007) L09002.

[14] J. V. Koski, T. Sagawa, O. Saira, Y. Yoon, A. Kutvonen, P. Solinas, M. Möttönen, T. Ala-Nissila, and J. P. Pekola, Nat. Phys. 9, 644 (2013).

[15] J. V. Koski, V. F. Maisi, T. Sagawa, and J. P. Pekola, Phys. Rev. Lett. 113, 030601 (2014).

[16] J. V. Koski, V. F. Maisi, J. P. Pekola, and D. V. Averin, Proc. Natl. Acad. Sci. USA 111, 13786 (2014).

[17] O.-P. Saira, Y. Yoon, T. Tanttu, M. Möttönen, D. V. Averin, and J. P. Pekola, Phys. Rev. Lett. 109, 180601 (2012). 
[18] J. P. Pekola, A. Kutvonen, and T. Ala-Nissila, J. Stat. Mech. (2013) P02033.

[19] M. Esposito and C. Van den Broeck, Phys. Rev. Lett. 104, 090601 (2010).

[20] D. V. Averin and K. K. Likharev, J. Low Temp. Phys. 62, 345 (1986).

[21] J. P. Pekola and O.-P. Saira, J. Low Temp. Phys. 169, 70 (2012).
[22] D. V. Averin and J. P. Pekola, Europhys. Lett. 96, 67004 (2011).

[23] O.-P. Saira, M. Möttönen, V. F. Maisi, and J. P. Pekola, Phys. Rev. B 82, 155443 (2010).

[24] E. Aurell and R. Eichhorn, New J. Phys. 17, 065007 (2015).

[25] J. Ankerhold and J. P. Pekola, Phys. Rev. B 90, 075421 (2014).

[26] R. Schmidt, M. F. Carusela, J. P. Pekola, S. Suomela, and J. Ankerhold, Phys. Rev. B 91, 224303 (2015). 